\title{
Grenzen des Sprachspiels im Dada
}

Mitten im ersten Weltkrieg, im Jahr 1916, öffnete sich in Zürich die Tür des ,Cabaret Voltaire' und das, was sich schon lange anbahnte, bekommt einen Ort: den Club ,Dada‘. „Es lebe das Chaos“ titelt am 29. Januar 2016 SPIEGEL-Online einen Artikel zum 100-jährigen Jubiläum dieser Kunstrichtung. Für wenige Jahre fasste der Dadaismus in den westlichen Metropolen Fuß. Zu der um dieselben Jahre herum geborenen Generation ihrer führenden Theoretiker und literarischen Praktiker zählten unter anderem in Berlin/Hannover Kurt Schwitters (1887-1948) und in Zürich Hugo Ball (1886-1927). ${ }^{1}$ Sie treiben ihre lyrischen Sprach- und Wortspiele innerhalb der sprachlichen Paradigmen so weit wie es nur geht, überschreiten schließlich die Textualität und die Sprachlichkeit bei ihren ästhetischen Spielen und erreichen damit systematisch gesehen einen experimentellen Endpunkt, der nicht mehr erlaubt, ohne weiteres vom Sprachspiel zu reden.

Deklamatorisch brachten die Dadaisten das Gegenprogramm zur traditionellen Strukturästhetik und bürgerlichen Autorästhetik ihrer Zeit auf den Punkt. Das hatte praktische Produktionsfolgen. Die Welt war im Schrecken des Weltkriegs aus den Fugen gegangen und nun auch die alte Kunstdoktrin. Die Prioritäten wurden umgestellt. Die klassizistischen ästhetischen Ideale der klischierten Formergebenheit des 19. Jahrhunderts waren erledigt. Nun triumphierte die ,Karawane‘ (von Hugo Ball) gewissermaßen als Schlüsseltext neuer ästhetischer Prinzipien, die darauf ausgerichtet waren, den bekannten Aufbau irgendeiner herkömmlichen Semantik zu verunmöglichen.

Die große dadaistische Dekonstruktion setzte auf literarischem Feld bei den sprachlichen Konventionen an und betrieb die Auflösung in einem breiten Spektrum. Es reicht von spielerischen Parodien bis hin zur totalen Amorphisierung sprachlichen Materials. Hier zwei Beispiele von Kurt Schwitters. In seinem angeblich „aus dem Chinesischen“ stammenden Gedicht ,Banalitäten“ (Abb. 1) spielt er parodistisch mit Allerwelts-Apophthegmata wie „Lügen haben kurze Beine“, „Eile mit Weile“, „Wie der Anfang, so das Ende“, „In der Kürze liegt die Würze“ oder dem Mephisto-Spruch aus dem zweiten Akt des ,Faust': „Wer kann was Dummes, wer was Kluges denken, das nicht die Vorwelt schon gedacht?“ Solche Banal-Lehrsätze aus dem Weisheitskästchen des Spießbürgers werden umformuliert, untereinander permutiert und mit teils tautologischen Zwischen-

1 Biographien und „Dada-Geographie“ in Puff-Trojan und Compagnon (2016: 155-173). 
versen kommentiert und aktualisiert (etwa mit Bezug auf den „Expressionismus“). So sollen die ,chinesischen“ (also in Wahrheit unverständlichen) Herrschafts-Topoi der Bourgeoisie bloßgestellt werden.

Banalitäten

aus dem Chinesischen

Fliegen haben kurze Beine.

Eile ist des Witzes Weile.

Rote Himbeeren sind rot.

Das Ende ist der Anfang jeden Endes.

$-$

Der Anfang ist das Ende jeden Anfangs.

Banalität ist jeden Bürgers Zier.

Das Bürgertum ist aller Bürger Anfang.

-

Bürger haben kurze Fliegen.

Würze ist des Witzes Kürze.

Jede Frau hat eine Schürze.

$-$

Jeder Anfang hat sein Ende.

Die Welt ist voll von klugen Leuten.

$-$

Kluge ist dumm.

-

Nicht alles, was man Expressionismus nennt, ist Ausdruckskunst.

Kluge ist immer noch dumm.

-

Dumme ist klug.

Kluge bleibt dumm.

Abb. 1: Kurt Schwitters: Banalitäten (in Riha und Schäfer 2015: 163-164) 
Im Gedicht ,Cigarren“ (Abb. 2) wird das im bürgerlichen Leben der Zeit beliebte Spiel mit Anagrammen so dekonstruiert, dass keine Sinneinheiten mehr bestehen bleiben, sondern nur noch kontingente Folgen von Graphemen (,elementar"), die sich auch nur noch mit Schwierigkeiten als klanglich fließende Lautfolgen skandieren lassen.

Cigarren (elementar)

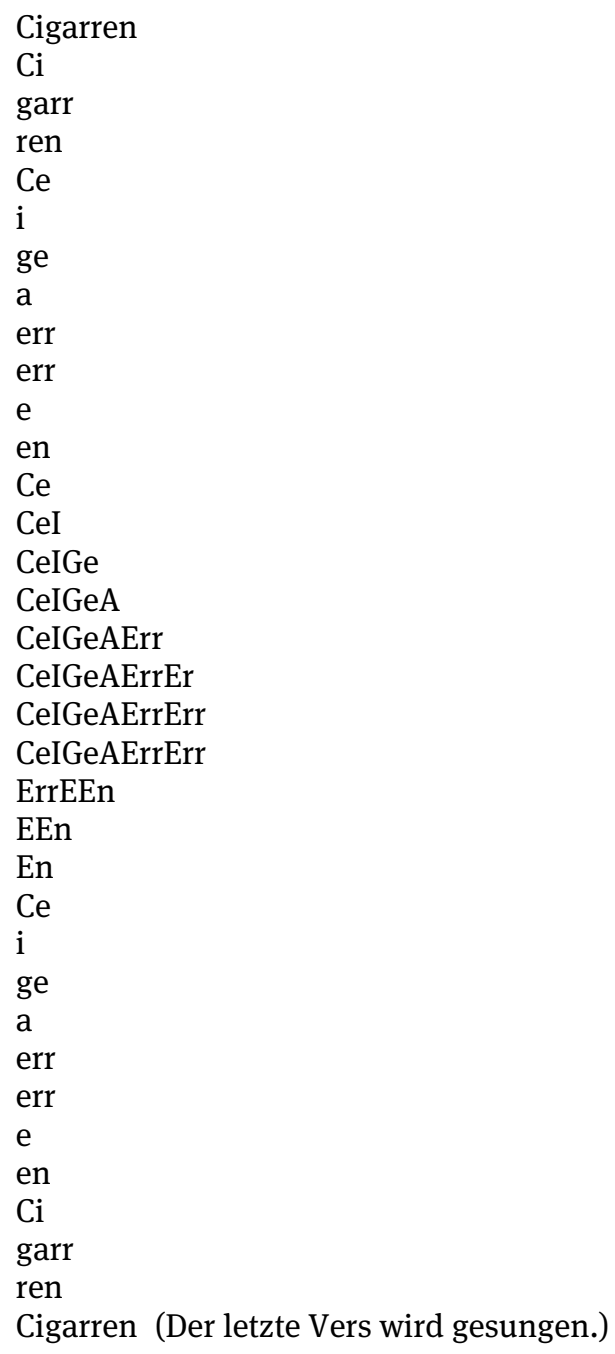

Abb. 2: Kurt Schwitters: Cigarren (elementar) (in Riha und Schäfer 2015: 165) 
Mit dem Wort Chaos ist das Lebensgefühl der Dadaisten-Generation auf den Begriff gebracht, für die in der Weltkriegskatastrophe die alteuropäischen Ordnungen aller Art endgültig zusammenbrachen. Den Zufluchtsort Zürich empfanden die vielen Immigranten und Exulanten, die sich dort im Krieg zusammenfanden, als Spiegel des ambivalenten, keineswegs bloß negativen Chaos-Gefühls der Zeit. „Das Vorkriegs- und Kriegs-Zürich war ein glänzendes Chaos“, schreibt ein Zeitzeuge; ähnlich Richard Huelsenbeck 1964: „Ich liebte das Chaos der Zeit“. ${ }^{3}$ Schon 1920 hatte Huelsenbeck die Erklärung des Clubs Dada aufgegriffen: „Dada ist Chaos, aus dem sich tausend Ordnungen erheben, die sich wieder zum Chaos Dada verschlingen.“ Die Dadaisten des Jahres 1916 waren Menschen, „die im Zustand der anomischen Unsicherheit das Chaos in sich erlebten“ (Huelsenbeck 1964: 9). In den frühen Programmschriften des Dada wird dieser Antagonismus von Ordnung und Chaos immer wieder beschworen, wobei Ordnung als alte Ordnung grundsätzlich in Frage gestellt wird (Huelsenbeck 1964: 103). Für Dadaisten ist das ein Akt der modernen Erlösung der Kunst. Ein aus einer Katastrophe erwachsenes Lebensgefühl wird als Prinzip für die Artefaktproduktion umformuliert. Programmatisch geht es den Dadaisten um eine neue Art des „Erlebens“, die „das Bedürfnis nach Ruhe und Ordnung“ überwindet (Huelsenbeck [1920] 1980: 7).

Was aber heißt es, programmatisch Chaos statt Ordnung im Text zu stiften? Raoul Hausmann gibt uns einen Hinweis, wenn er $\mathrm{zu}$ beobachten meint, dass Deutschland „von westlichen Formeln zu östlicher Formlosigkeit“ gedriftet sei (Hausmann [1920] 1980: 148). Die Idee der „Formlosigkeit“ kann bei der Textproduktion aber sehr weit gehen. Es kommen Produktionsprinzipien ins Spiel, die uns beinahe überraschend an Theoreme des so genannten postmodernen Denkens im ausgehenden 20. Jahrhundert denken lassen. So stellt Tristan Tzara in seinem ,Manifest Dada 1918“ das logische Gesetz der Widerspruchsfreiheit in Frage und postuliert eine auf Paradoxien gebaute Fundamentalambiguität für die Kunst, nämlich, „daß man mit einem einzigen frischen Sprung entgegengesetzte Handlungen gleichzeitig begehen kann; ich bin gegen die Handlung; für den fortgesetzten Widerspruch, für die Bejahung und bin weder für noch gegen“ (Tzara in Riha und Schäfer 2015: 36). - „Dada bedeutet nichts.“ und Dada entsteht aus einer Abkehr vom Konventionellen, einer Haltung „des Mißtrauens gegen die Gemeinsamkeit“ (Tzara in Riha und Schäfer 2015: 36-37). Das hat Folgen für die Nutzung der Sprache (la langue) als dem tragenden konventionellen System in Kulturen schlechthin. Gemeinsame Verständigungs-

2 Fritz Brupbacher 1930 im Rückblick. Zit. n. Behrens (2016: 211, Hervorhebung von mir).

3 Huelsenbeck: Dada oder der Sinn im Chaos. In Huelsenbeck (1964: 7-23, hier: 21). 
systeme werden suspekt: Das „annehmbarste System ist das, grundsätzlich keines zu haben“ (Tzara in Riha und Schäfer 2015: 41). Damit kommen alle sozialen Denkkonventionen ins Wanken: „Logik ist immer falsch. Sie zieht die Begriffe am Faden, Worte, in ihrer formellen Äußerlichkeit, hin zu den Enden illusorischer Mittelpunkte. Ihre Ketten töten, gewaltiger Tausendfuß, erstickt die Unabhängigkeit. Mit der Logik vermählt würde die Kunst im Incest leben“ (Tzara in Riha und Schäfer 2015: 43). Diese Abkehr von logischen Strukturen taucht dann wieder programmatisch bei postmodernen Theoretikern wie Derrida oder Johnston auf (Knape 2015).

Als revolutionär muss auch die neue dadaistische Collage-Technik angesehen werden, die die alten Gattungen auflöst und die Trennung der semiotischen Systeme aufhebt. Sie wurde in Gemälden, Objekt-Bild-Kompositionen und FotoCollagen umgesetzt. Dabei werden nicht nur die Bildzeichen, die Schriftzeichen, übliche Textstrukturen und der gesamte kulturelle Symbolvorrat zerhackt, neu kombiniert und in eine chaotische Gemengelage gebracht, sondern auch die Layout-Formate (also etwa das klar abgegrenzte Quadrat oder Rechteck des Bildes) zerstört. Über allem steht das bewusst gewählte Wort „Chaos“. Nicht nur die Rekonstruktion der Bedeutungen im linguistischen Sinn führt ins Chaos, sondern auch die Suche nach ästhetischen Ordnungsmodellen. Alles ist aufgelöst. Nach 100 Jahren Dada wissen wir, dass sich dieses Loslassen konventioneller Regelwerke vor allem in der bildenden Kunst immer weiter verbreitete, während Dada in der Literatur eher nur eine kurze Phase war. In der Malerei war jetzt das Tor zur reinen Abstraktion geöffnet. Das hatte sich längst schon im Impressionismus angedeutet, doch nun gab es kein Halten mehr. Damit wird der Übergang von der strikten ästhetischen Ordnung zum Chaos und umgekehrt vom vermeintlich chaotischen Faktor des künstlerischen Materials hin zu neuen Kunstordnungen zu einem Signum der Moderne.

Berühmt geworden sind Hugo Balls erstmals 1917 unter dem Titel ,Karawane' veröffentlichte Buchstabenkombinationen (Abb. 3 und 4). ${ }^{4}$ Wir sehen eine Aneinanderreihung von graphischen Elementareinheiten auf Grundlage des lateinischen Alphabets. Aber ist es auch ein Text? Zweifellos ist es programmatisch und markiert ein Artefakt, das man als ästhetisches Objekt bezeichnen muss. Die Herausgeber der Werke Hugo Balls sehen das anders. Sie haben die ,Karawane“ unter dem Rubrum „Gedichte“ veröffentlicht und damit einer ganz bestimmten, eingeführten Textsorte zugeordnet (Hugo Ball: Gedichte, S. 68). Für uns stellt sich angesichts dessen die Frage, ob es tatsächlich ,Texte، ohne ge-

4 In Originalreproduktion mit den verschiedenen Drucktypen dieses historischen (nicht autorisierten) Druckes wiedergegeben bei Huelsenbeck ([1920] 1980: 53). 
prägte semantische Einheiten gibt? Kann es ein Bedeutungsgebäude ohne semantisch geladene Bausteine geben? Die Antwort lautet Nein. Den Beweis erbringt das Misslingen jeden Versuchs, ein Close Reading (eine Art literarischen Ekphrasis-Tests) durchzuführen. ${ }^{5}$ Unter linguistischer Perspektive enthält dieses ästhetische Objekt - vom Titelwort einmal abgesehen - nämlich keine einzige segmentale, klar erkennbar geprägte Wort-Bedeutungseinheit, nur Schriftelemente. Was bei Schwitters' ,Banalitäten' noch der entscheidende Semantik-Trigger war, nämlich die durchgängig erhaltenen geprägten semantischen Bausteine, wird bei Hugo Ball an die Grenze geführt, und damit zugleich der Kern des Textualitätsverständnisses. $\mathrm{Zu}$ den asemantischen Buchstabenfolgen fügen sich neben dem Wort in der Überschrift („Karawane“) nur noch einige wenige entfernte Anklänge an deformierte Wörter, die teils wie ein Bezug auf andere Sprachen klingen (etwa jolifanto - Elefant, bambla - trampeln, grossiga - groß, russula - Rüssel). Hingegen taucht kein einziger grammatischer Satz auf, der als Exemplar der nächst höheren Ebene bedeutungstragender Bausteine von lautsprachlichen Texturen gelten könnte.

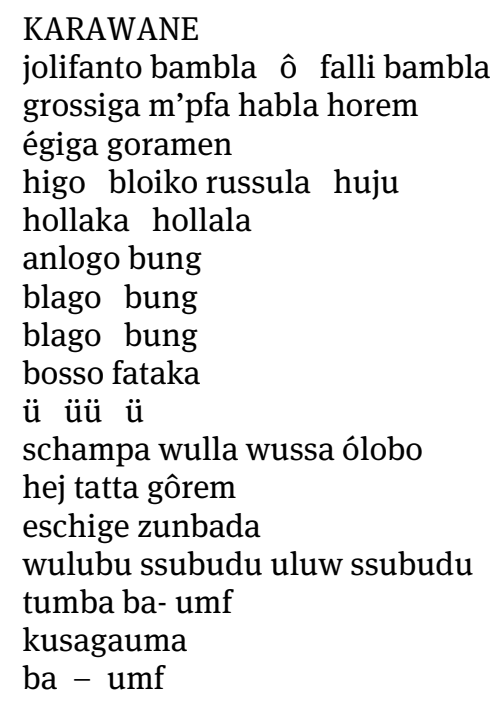

Abb. 3: Hugo Ball: Karawane (in Riha und Schäfer 2015: 55)

5 Der Ekphrasis-Test ist eine Metabeschreibung visueller Eindrücke mittels Lautsprache. Diese Methodenvariante kann man bei der semantischen Analyse von Objekten mit Code-Mix anwenden. Die Ekphrasis hilft uns, auf dem Wege der Transnotation die Semantik visueller Objekte (zumindest als denotative Kondensate) zu ermitteln. Siehe Knape (2016: 92). 


\section{KARAWANE}

jifanto bambla ô falli bambla

grossiga mipfa habla horem

égiga goramen

higo bloiko russula huju

hollaka hollala

anlogo bung

blago bung

blago bung

bosso fataka

a

schampa wulla wussa ólobo

hej tatta gôrem

eschige zunbada

wulubu Ssubudu uluw ssubudu

tumba ba- umf

kusagauma

ba - umf

Hugo Ball

Abb. 4: Original-Typographie und -Layout von Hugo Balls 'Karawane’. Abb. nach Huelsenbeck ([1920] 1980: 53)

Wenn dies kein Text ist, was ist das Gebilde aus bloßen Graphem-Aneinanderreihungen aber dann? Das Fehlen der beiden zentralen Wurzel-Konstituenten von Text, nämlich a) semantisch geprägter Bausteine und b) ihrer geordneten Relationen führt uns bei der Analyse auf ein ganz anderes Terrain. Dazu gibt uns die genannte Ball-Werkausgabe einen Hinweis. Sie ordnet die ,Karawane“ 
einer ,Gedicht“-Gruppe mit dem Namen „Lautgedichte“ zu. Darin kann man einen Hinweis sehen, die Diskussion über den Charakter dieses Artefakts zunächst einmal auf phonologischer oder phonetischer bzw. auch auf suprasegmentaler Ebene zu führen. Läge hier ein klarer Fall von Onomatopoie vor, kämen unter Umständen analoge Bedeutungsprägungen der Elemente vor, wie wir sie bei der Nachahmung von Tierlauten kennen. Dass Hugo Ball noch einen anderen Titel der Karawane vorsah, könnte in diese Richtung führen. Er nannte es auch „Zug der Elefanten“ (in Hugo Ball: Gedichte, S. 68). Freilich ist es problematisch, den Titel als Aufführungsanleitung zu verstehen, denn diese Lautfolge ahmt kaum die Geräusche des Trampelns einer Elefantengruppe wirklich lautmalerisch nach. Dennoch ist der Gedanke an eine Partitur nicht ganz abwegig. Die Skansion bzw. der laute Vortrag und die Intonation der erkennbaren Phoneme und Phonemgruppen war beabsichtigt, wohl aber nicht die genaue akustische Elefantenimitation.

Damit sind wir an der Grenze der Linguistik angelangt und überschreiten sie in Richtung Tonsemantik der Musik sowie Semantik weiterer erlernter GeräuscheCodes. Deren kleinste Bedeutungseinheiten sind aber nicht mit den Wörtern/Zeichen der Lautsprache und des BildCodes zu verwechseln. Die Musik hat kein universales Lexikon, sondern im Bereich der westlichen Musik nur kodifizierte (semantikfreie) Töne, die nach diversen Harmonien geordnet werden können.

Hugo Ball steht mit seiner Hinwendung zur Agogik und zur musikalischen Partitur nicht allein. Bekannt geworden ist vor allem auch die ,Ursonate، von Kurt Schwitters aus dem Jahr 1925, die mit Satzbezeichnungen aus der Musik arbeitet (z. B. „Largo“) und ähnliche Lautfolgen aufweist wie Hugo Balls ,Karawane' (Faksimile bei Dietrich 2006: 172). Doch dabei geht es nie um die musikalischen Tonfolgen, die man bei Verwendung lateinischer Buchstaben mit do, re, mi, fa, sol, la, si, do notieren müsste. Nein, hier geht es um einen anderen Code. Die Dadaisten Ball und Schwitters schaffen Partituren zum Aufführen von Lauten des indogermanischen Lautsystems und nehmen dazu Schriftzeichen aus dem Alphabet. Damit ergibt sich wenigstens ein Restbezug zur Sprachlichkeit im engeren Sinn. Im vorliegenden Fall ist auf die Frage der Eigenständigkeit der systematischen Ebene der Phonologie und Phonetik mit ihren Möglichkeiten unscharfer Semantisierung unter extrem suprasegmentalen ,Laborbedingungen' (die ausnahmsweise ohne das sonst immer gegebene segmentale Sprachfundament arbeiten) $\mathrm{zu}$ verweisen, denn hier zeigen sich Verallgemeinerungsmöglichkeiten besonderer Art.

Balls grafisches Cluster eröffnet insgesamt den Blick auf diverse semiotische Felder, die differente Begriffe für das Problemfeld anbieten: Phonetik (Lin- 
guistik), Colorik (Malerei), Layout (Typographie), Agogik (Musik), Akustik (Geräuschkunde) usw. An dieser Stelle wechseln wir nun endgültig von der Textheorie auf andere Gebiete, etwa auf den Bereich der akustischen Ästhetik und auf die Ebenen der Medialisierung und der visuellen Ästhetik. Wie die Abbildung des Original-Layouts zeigt (Abb.4), spielt Hugo Ball auch mit der Typographie und dem Layout der Druckseite. Hier tut sich so etwas wie performative Ambiguität auf. Das Objekt schwankt unentscheidbar zwischen akustischer und optischer Performanz. Sind es nur einzelne Grapheme oder doch Notate von aufführbaren Laufolgen (freilich ohne sprachlichen Sinn)? Soll die Lautfolge überhaupt akustisch performiert werden? Die Herausstellung der Typographie im Originaldruck lenkt auf das rein optische Terrain. Damit kippt die ,Karawane‘ in Richtung eines grafischen Objekts. Es nähert sich dem an, was man später in der Kunst Lettrismus genannt hat (Grafiken aus Buchstaben). ${ }^{6}$ Sind diese visuellen Objekte des Dada aber noch Texte?

Der auffällige Wechsel der Drucktype von Zeile zu Zeile erzeugt ein besonderes ästhetisches Erleben, das aber als reine Typographiefrage systematisch gesehen nichts mit Lautsprache oder gar mit Textualität zu tun hat. ${ }^{7}$ Hinweise zum Aufbau einer Ordnung des Gesamtobjekts finden wir nur, wenn wir die Ebene der Textheorie verlassen und uns den Medialisierungsbedingungen, konkret: dem Layout, zuwenden. Die europäischen Konventionen des DruckLayouts von Gedichten, mit Spatien zwischen den Graphemen, der Absetzung von Zeilen und graphischen Trennelementen (bei der ,Ursonate' von Schwitters) können wir vielleicht als quasi-syntaktische Einteilungsformen verstehen. Die ,Karawane' bekommt aufgrund des Druckbildes in unserer Wahrnehmung den Charakter einer medial ,simulierten“ Textur. Man hat solche und ähnliche Gedichte auch konkrete Poesie genannt und damit das Übergängige hin zur bildenden Kunst und $\mathrm{zu}$ ästhetischen Artefakten sui generis markiert. Die texttheoretische Betrachtungsweise löst sich hier zugunsten einer anderen, der objektästhetischen Sicht auf. Dennoch ist die Diskussion solcher Fälle äußerst erhellend, weil sich in Diskussionen über Grenzen der theoretische Kern von Modellierungen deutlicher zeigt.

6 Ein frühes Beispiel von Tristan Tzara (aus dem Jahr 1916) in Puff-Trojan und Compagnon (2016: 37).

7 Man kann hier höchstens an den eventuell vorliegenden, schon erwähnten Rekurs auf das reine Phoneminventar des Deutschen bzw. die entsprechenden Grapheme beim Notieren deutscher Texte denken. 


\section{Literaturangaben}

Behrens, Nicola. 2016. Revolte. In Genese Dada. 100 Jahre Dada Zürich. Katalog zur Ausstellung im Arp Museum Bahnhof Rolandseck. Remagen 14. Februar bis 10. Juli 2016, 209212. Zürich: Scheidegger \& Spiess.

Dietrich, Dorothea. 2006. Hannover. In Leah Dickerman (Hg.), DADA. Zurich - Berlin - Hannover - Cologne - New York - Paris. Catalog National Gallery of Art Washington, 154-213. London \& New York: D.A.P. / The National Gallery of Art, Washington.

Hausmann, Raoul. [1920] 1980. Rückkehr zur Gegenständlichkeit in der Kunst. In Huelsenbeck [1920] 1980, 147-151.

Huelsenbeck, Richard. [1920] 1980. Poetische Aktion. Dada Almanach. Berlin 1920, Reprint Hamburg: Verlag Lutz Schulenburg.

Huelsenbeck, Richard (Hg.). 1964. Dada. Eine literarische Dokumentation. Reinbek bei Hamburg: Rowohlt.

Hugo Ball: Gedichte. Hg. v. Eckhard Faul. Göttingen: Wallstein, 2007 (= Hugo Ball. Sämtliche Werke und Briefe 1).

Knape, Joachim. 2015. Inversive Persuasion. Zur Epistemologie und Rhetorik der , Rhetorik der Verunsicherung'. In Ramona Früh et al. (Hgg.), Irritationen. Rhetorische und poetische Verfahren der Verunsicherung, 5-60. Berlin etc.: De Gruyter.

Knape, Joachim. 2016. Was ist ein Bild? Ein Kunstgespräch im Atelier Friedrich mit dem anwesenden Herrn Goethe. Wiesbaden: Harrassowitz.

Puff-Trojan, Andreas \& H.M. Compagnon (Hgg.). 2016. dada Almanach. Vom Aberwitz ästhetischer Contradiction. Textbilder. Lautgedichte. Manifeste, 155-173. Zürich: Manesse.

Riha, Karl \& Jörgen Schäfer (Hgg.). 2015. DADA total. Manifeste, Aktionen, Texte, Bilder. Stuttgart: Reclam.

Tzara, Tristan. Manifest Dada 1918. In Riha \& Schäfer (Hgg.), 35-45.

Prof. Dr. Joachim Knape ist seit 1991 Rhetorikprofessor am Seminar für Allgemeine Rhetorik der Eberhard Karls Universität Tübingen; seit 2018 hat er dort eine Seniorprofessur inne. Seine Forschungsgebiete umfassen die Theorie und Geschichte der Rhetorik, rhetorische Semiotik, Medienrhetorik, Textrhetorik, Rhetorik der Bilder, Poetik und Ästhetik sowie Renaissance Studien und Deutsche Sprache und Literatur. 